\title{
Intergenerational metabolic priming by sperm piRNAs
}

2 Adelheid Lempradl ${ }^{1,2, *}$, Unn Kugelberg ${ }^{3, \dagger}$, Mary Iconomou ${ }^{1, \dagger}+$, Ian Beddows ${ }^{2}$, Daniel Nätt ${ }^{3}$, 3 Eduard Casas ${ }^{4}$, Lovisa Örkenby ${ }^{3}$, Lennart Enders ${ }^{1}$, Alejandro Gutierrez Martinez ${ }^{1}$, Oleh 4 Lushchak $^{5}$, Erica Boonen ${ }^{1,2}$, Tamina Rückert ${ }^{1}$, Martin Sabev ${ }^{1}$, Marie G.L. Roth ${ }^{3}$, Dean Pettinga ${ }^{2}$, $5 \quad$ Tanya Vavouri ${ }^{4}$, Anita Öst ${ }^{3}, \uparrow^{*}$, J. Andrew Pospisilik ${ }^{1,2, \dagger}$

$6 \quad{ }^{1}$ Max-Planck Institute for Immunobiology and Epigenetics, Stuebeweg 51, 79108 Freiburg, 7 Germany

$8 \quad{ }^{2}$ Van Andel Research Institute, 330 Bostwick Ave. N.E., MI 49503, USA, current address for 9 AL and JAP

$10{ }^{3}$ Department of Biomedical and Clinical Sciences, Linkoping University, 58183 Linkoping, 11 Sweden

$12{ }^{4}$ Josep Carreras Leukaemia Research Institute (IJC), Campus ICO-IGTP-UAB, Ctra. de Can

13 Ruti, Camí de les Escoles s/n, 08916 Badalona, Barcelona, Spain

$14{ }^{5}$ Vasyl Stefanyk Precarpathian National University, 57 Shevchenko Str., Ivano-Frankivsk, 15 76018, Ukraine

$16 \uparrow$ These authors contributed equally

$17 \$$ These authors contributed equally

18 *Correspondence to: anita.ost@liu.se and heidi.lempradl@vai.org 
20 Summary: Preconception parental environment can reproducibly program offspring phenotype

21 without altering the DNA sequence, yet the mechanisms underpinning this 'epigenetic inheritance'

22 remains elusive. Here, we demonstrate the existence of an intact piRNA-pathway in mature

23 Drosophila sperm and show that pathway modulation alters offspring gene transcription in a

24 sequence-specific manner. We map a dynamic small RNA content in developing sperm and find

25 that the mature sperm carry a highly distinct small RNA cargo. By biochemical pulldown, we

26 identify a small RNA subset bound directly to piwi protein. And, we show that piRNA-pathway

27 controlled sperm small RNAs are linked to target gene repression in offspring. Critically, we find

28 that full piRNA-pathway dosage is necessary for the intergenerational metabolic and

29 transcriptional reprogramming events triggered by high paternal dietary sugar. These data provide

30 a direct link between regulation of endogenous mature sperm small RNAs and transcriptional

31 programming of complementary sequences in offspring. Thus, we identify a novel mediator of

32 paternal intergenerational epigenetic inheritance. 


\section{Introduction}

35 Current data suggest obesity as one of the world's chief socioeconomic challenges of our day, impacting $\sim 1$ billion individuals worldwide. The dramatic rise in metabolic disease incidence in

37 the last decades, particularly in children, suggests a prominent role for epigenetic mechanisms, in

38 particular, a role for intergenerational epigenetic mechanisms where physiological effects in parents (e.g. diet, hyperglycemia, obesity) trigger disease-predisposing shifts in the offspring through non-DNA-sequence-based mechanisms. To date, the mechanisms mediating intergenerational epigenetic programming in response to physiological state remain poorly understood.

44 PIWI-interacting RNAs (piRNAs) are 24-32 nucleotide, PIWI-bound small RNAs that are best 45 known for silencing transposable elements and thereby limiting their mutagenic potential ${ }^{1-3}$. This canonical function of piRNAs is essential for germline genome integrity and is active in most animals ${ }^{4}$. The piRNA-pathway differs from other small RNA (sRNA) pathways (miRNA, siRNA) in three key aspects: 1) piRNA-pathway protein expression is mainly restricted to reproductive organs; 2) piRNAs are generated through a Dicer-independent mechanism; and 3) piRNAs are processed from single stranded precursor transcripts, making mRNAs theoretical sources and substrates for piRNA generation and amplification.

Canonical piRNAs are derived from genome regions called piRNA clusters, which harbor ancient transposon fragments. Accumulating evidence in both flies and mice suggests that piRNAs can also be produced from genic mRNAs ${ }^{5-10}$. In male mice, about $20 \%$ of the piRNA population in pre-pachytene germ cells for example, is derived from the exons of hundreds of mRNAs ${ }^{5}$. piRNAs derived from 3'UTRs have also been found in follicle cells of fly ovaries and Xenopus eggs ${ }^{6,7,10}$. Although piRNAs were first identified in Drosophila testis ${ }^{11}$, much of the pioneering work in flies has focused on the female germline ${ }^{12}$. In general, the sRNA content of the male Drosophila 60 germline is ill characterized. Specifically, piRNA populations, their dynamics during spermatogenesis and their potential functional roles, remain poorly understood.

63 In C. elegans, piRNAs have been implicated in the multigenerational inheritance of foreign DNA64 triggered epigenetic silencing ${ }^{13}$. In these contexts, once piRNA-seeded silencing states are initially 
65 established, their long-term memory is independent of the original piRNA trigger, and instead

66 relies on nuclear RNAi and chromatin pathways. Notable, maternal piRNAs have been shown to

67 buffer against DNA sequence incompatibility between maternal and paternal genomes, in

68 particular at transposons, a phenomenon known as hybrid dysgenesis ${ }^{14}$. Here, we identify an intact

69 mature sperm piRNA pathway and provide evidence for its involvement in both encoding and

70 decoding intergenerational inheritance effects.

72 Dynamic sRNA expression in the male Drosophila germline

73 The tube-shaped Drosophila testis has contributed significantly to our understanding of stem cell

74 maintenance and germ cell differentiation ${ }^{15}$. To understand the dynamics of sRNA populations

75 during spermatogenesis we performed sRNA sequencing on testes manually dissected into four

76 parts: 1) testis tip (T1) containing stem cells and primary spermatocytes, 2) the apical portion (T2)

77 containing meiotic and developing spermatogonia, 3) the distal portion (T3) containing late-stage

78 spermatocytes undergoing individualization, and 4) mature sperm (Sperm) isolated from seminal

79 vesicles (Fig. 1A-C). Principal component analysis revealed high technical reproducibility.

80 Interestingly, the greatest variation (PC1) separated sperm from testis samples; PC2 separated the

81 three stages of testis development (Fig. 1B). These data indicate that the mature sperm sRNA

82 repertoire is highly distinct from that of the developing germ cells. Relative to T1-T3, sperm

83 showed an increase in sRNAs mapping to protein coding genes (3' UTRs and exons) and tRNAs,

84 and a decrease in repeat-associated sRNAs (piRNA clusters, complex and simple repeats) (Fig.

85 1C). These results resemble findings in the male mouse germline, where a loss of piRNAs and a

86 gain of tRNA and mRNA fragments was shown for the transition from testicular to caput sperm ${ }^{16}$.

87 Sperm sRNAs mapping to protein coding exons exhibited a high correlation with sperm mRNA-

88 seq datasets $(r=0.92, p<0.0001$; Fig. 1D), consistent with the mRNA degradation that occurs in

89 late spermatogenesis. Fly and human sperm ${ }^{17}$ sRNA (Fig. 1E and F) repertoires showed critical

90 similarity at the biotype level, sharing for instance seven of the ten most highly expressed tRNA

91 genes (Fig. 1G), thus highlighting the evolutionary conservation. These results comprise the first

92 in-depth analysis of sRNA dynamics in Drosophila spermatogenesis; they demonstrate a highly

93 specific sperm sRNA population relative to the developing germline; and, they show conservation

94 in sRNA composition between Drosophila and mammals. 


\section{Evidence for a functional piRNA-pathway in mature sperm}

97 One key outstanding question about sperm sRNAs in the context of intergenerational control is

98 how the minimal RNA load of a single sperm cell can reprogram the developmental trajectory of

99 a whole organism. The amplification mechanism of the piRNA-pathway provides one possibility

100 by which quantal differences in sRNAs might trigger reproducible next-generation effects as well

101 as a range of penetrance distributions. piRNAs were originally identified and characterized based

102 on their direct binding to PIWI proteins ${ }^{18-22}$. If piRNAs were involved in epigenetic inheritance,

103 we reasoned that they would be loaded onto the appropriate protein machinery in mature sperm.

104 Previous work showed that Piwi protein is stably expressed in somatic and early germ-cells in the 105 apical testis ${ }^{23}$. Consistent with those reports, we found Piwi immunoreactivity in the nuclei of 106 multiple germline and somatic cell types in the tip of the testis (Supplemental Fig. 1A). 107 Interestingly, we also found piwi protein in individualizing, late stage spermatids at the distal end 108 of the testis, and in washed and purified sperm isolated from the sperm sack when using anti-Piwi 109 antibody raised against a peptide from the middle of the protein (Fig. 2A left; see Materials and 110 Methods for antibody details;). Piwi immunoreactivity appeared both as puncta along the sperm 111 tail (Fig. 2A center) and as a highly reproducible single body at the base of each compacted sperm 112 nucleus (Fig. 2A right, and secondary antibody control in Supplemental Fig. 1B). This result is in 113 agreement with results from mouse showing PiwiL1 protein presence in mature sperm ${ }^{24}$. Western

114 blots confirmed our result by detecting the full-length Piwi protein in isolated sperm and ovaries 115 ( 97 kDa; Western blot, Fig. 2B left and middle panel). Samples containing empty seminal 116 vesicles showed no evidence of Piwi protein, which argues against possible contribution of somatic 117 cell contamination to the signal in sperm. Further, we isolated protein lysates from ovaries, testis, 118 and purified sperm of flies carrying a BAC Piwi-GFP transgene. In contrast to wild-type, Western 119 blot of Piwi-GFP transgenic sperm revealed two bands, matching the sizes of GFP-tagged and 120 wild-type Piwi protein (Fig. 2C). Thus, both wild-type and transgenic Piwi protein are found in 121 sperm, confirming the specificity of the antibody.

123 Seeking to leverage an antibody-independent technique, we performed targeted mass spectrometry 124 on protein extracts of purified sperm (Supplemental Fig. 1C). For Piwi, we found signals for all 125 possible targetable Piwi peptides after tryptic digestion. Importantly, peptides were also detected 126 for the remaining two PIWI proteins known to be necessary for amplification of silencing 
127 competent piRNAs, namely aubergine (Aub) and argonaute 3 (AGO3)(Supplemental Fig. 1C).

128 Thus, intact PIWI pathway proteins are consistently expressed in mature sperm and may therefore serve as facilitators of intergenerational inheritance of functional piRNAs.

To identify piwi-associated sRNAs we used the sperm specific Piwi antibody to develop and

132 optimize a biochemical pulldown and sequencing approach (Piwi-RIP-seq) applicable to the

133 minute amounts of Piwi protein and RNA available in dissected Drosophila sperm. After rounds

134 of protocol optimization, we profiled the Piwi-bound sRNA content in sperm from 4 replicates

135 each of $\sim 750$ hand-dissected seminal vesicles (Piwi-RIP-seq). Piwi-pulldown enriched sRNAs

136 showed a peak length of $\sim 25 \mathrm{nt}$ (Fig. 2D left) and an A-bias at the first nucleotide position (Fig. 2D

137 right), consistent with published data from ovaries ${ }^{18}$. Using a stringent mapping and hierarchical

138 annotation approach and rRNA exclusion (see methods), we found enrichment for sRNAs from

139 piRNA clusters, repeats and transposons, and to a lesser extent those from protein coding exons,

140 5'UTRs and ncRNAs (Fig. 2F, top). By contrast, sRNAs not associated with piwi protein in the

141 pull-down experiment showed a peak length of $\sim 21-23 \mathrm{nt}$ (Fig. 2E) and were enriched for miRNAs,

142 tRNAs, snRNAs, snoRNAs pseudogenes and 3'UTRs (Fig. 2F, bottom). Importantly, Piwi-bound

143 RNAs exhibited an anti-correlation with total sRNA content of sperm (Fig. 2G). These data

144 indicated highly specific loading of sRNAs onto Piwi and also argued against non-specific

145 pulldown effects in the Piwi-RIP-seq results. Pathway analysis of protein coding exon-derived

146 sRNAs showed enrichments for genes involved in phosphorylation, metabolism, gametogenesis,

147 development and cilium organization (Fig. 2I). To test for potential chromatin state associated

148 enrichment we next mapped our Piwi-RIP-seq dataset to published Drosophila chromatin state

149 annotations derived from genome-wide binding analysis of over 50 functional chromatin-binding

150 proteins $^{25}$. Interestingly, this intersection showed a striking enrichment for sRNAs from transcripts

151 that map to chromatin states with clear repressive signatures. These enriched states were described

152 as Lamin / H1-associated, "Black" chromatin and Polycomb-associated, "Blue" chromatin in

153 embryonic cells (Fig. 2H and Fig S1 D). Thus, mature sperm harbors a highly specific complement

154 of Piwi-bound sRNAs (piRNAs).

156 Piwi controlled sperm RNAs regulate offspring gene transcription 
157 Working from the hypothesis that sperm piRNAs might regulate offspring transcription, we 158 examined the effect of parental heterozygous aub mutation $\left(a u b^{\text {Het }}\right)$ on next-generation 159 transcriptional output (embryos, stage 17). Aub participates in the ping-pong cycle of the PIWI 160 pathway and is necessary for amplification of silencing competent piRNAs and robust gene 161 repression ${ }^{26,27}$. We compared mRNA transcriptional changes elicited in offspring from two parallel 162 mutant crosses (Fig. 3A and B): $a u b^{\text {Het }}$ mothers x WT fathers (maternal $a u b^{\text {Het }}$ offspring); and WT 163 mothers $\mathrm{x} a u b^{\text {Het }}$ fathers (paternal $a u b^{\text {Het }}$ offspring)(data expressed relative to wildtype offspring 164 from parallel WT $\mathrm{x}$ WT crosses; WT offspring). In order to ensure comparable genetic 165 backgrounds, we prepared for the experiment by backcrossing $a u b^{\text {Het }}$ flies to our own highly inbred 166 w1118 background (WT) (see supplemental methods for details). The mRNA transcriptional 167 response in maternal $a u b^{\text {Het }}$ offspring revealed a strong correlation with our RIP-seq defined sperm piRNAs (Fig. 3C), specifically, derepression of piRNA-sequence matching 'targets'. These data suggested that wild-type Piwi-bound sperm RNAs directly or indirectly trigger silencing of

170 sequence-matching loci in the next-generation (zygote). This conclusion agrees with previous 171 findings that maternal depletion of Piwi impacts heterochromatin formation in the offspring ${ }^{28}$. In 172 reciprocal crosses, the paternal $a u b^{\text {Het }}$ mutation failed to trigger a similar piRNA dependent 173 offspring transcriptional response (Fig. 3D) indicating a parent-of-origin directionality to the 174 system. Thus, offspring transcription is modulated in a parent-specific manner by piRNA-pathway 175 dosage.

177 To gain further insights into the nature of parent-specific transcriptional changes in offspring, we 178 compared the changes triggered in maternal-aub $b^{\text {Het }}$ offspring to those of paternal-aub $b^{\text {Het }}$ offspring, 179 and identified three classes of intergenerationally-responsive transcripts and associated sRNAs:

180 Class I transcripts (Fig. 3B Purple; $\mathrm{n}=1046$ ) were upregulated in offspring of both maternal and 181 paternal $a u b^{\text {Het }}$ crosses $\left(\log _{2} \mathrm{FC}>0.5\right)$. Class I transcript sequence matching sRNAs were enriched 182 in our Piwi-RIP-seq indicating these transcripts are 'targets' of bona fide Piwi-bound sperm 183 piRNAs (Fig. 3F). Class I transcripts were enriched for transposons (Supplemental Fig. 2A) which 184 is in agreement with the canonical role of piRNAs in transposon silencing. Together, these data 185 indicate that Class I genes in the embryo are targets of paternal piRNAs and their repression in the 186 offspring requires zygotic aub activity. They identify a novel intergenerational sequence-specific 187 gene regulatory axis. 
188 Class II transcripts ( $\mathrm{n}=494)$, by contrast, were upregulated only in offspring of paternal aub $b^{\text {Het }}$ 189 crosses (Fig. 3B, Green; paternal $\log _{2} \mathrm{FC}>0.5$; maternal $\log _{2} \mathrm{FC}<-0.5$ ). sRNAs mapping to 190 Class II transcripts were depleted in the Piwi-IP (Fig. 3E) and, interestingly, were highly and 191 specifically expressed in sperm (Fig. 3G). Further experiments revealed that sRNAs mapping to

192 Class II transcripts were sensitive to both $a u b$ and piwi dosage in the male germline (reduced 193 expression in $a u b^{\text {Het }}$ (Fig. 3E) and in $p i w i^{H e t}$ (Supplemental Fig. 2B) sperm sRNAseq). These data 194 identify a novel subclass of highly expressed sRNAs in sperm whose abundance is sensitive to aub 195 and piwi dosage. Thus, Class II genes are intergenerationally regulated transcripts responsive to 196 paternally inherited, piRNA-pathway sensitive sRNAs.

197 Class III transcripts $(\mathrm{n}=1036)$ were down regulated in offspring of both maternal and paternal $198 a u b^{\text {Het }}$ crosses (Fig. 3B, Dark Grey; $\log _{2} \mathrm{FC}<-0.5$ ). The Class III genes had no detectable 199 signatures associated with transposon content (Supplemental Fig. 2A), Piwi-binding (Fig. 3F), or 200 piRNA-pathway sensitivity (Fig. 3E and Supplemental Fig. 2B). There were only 20 Class IV 201 transcripts that were specifically upregulated in maternal crosses (and only six with sequence202 matching sperm sRNA reads, Fig. 3B, Light Grey; paternal $\log _{2} \mathrm{FC}<-0.5$; maternal $\log _{2} \mathrm{FC}>$ 203 0.5). Importantly, to rule out potentially confounding influences of the arbitrary expression change 204 cutoffs used above we repeated the analysis using a threshold free stratified Rank-Rank 205 Hypergeometric Overrepresentation (RRHO) approach ${ }^{29}$ (Right side of Fig. S2). RRHO validated 206 the interpretations above and the existence of Class I, II, and III transcripts (Fig. S2C) and their 207 associated sRNA signatures (Fig. S2 D-F).

209 Gene set over-representation analysis showed enrichment in signaling and neuronal pathways (Fig. 210 S2G top) for Class I (purple) genes. Class II (green) genes were enriched for metabolic, hydrolase 211 activity and neuronal pathways (Fig. S2 middle), and Class III genes (dark gray) were enriched for 212 cell cycle pathways (Fig. S2G bottom) genes. Consistent with the enrichments observed in our 213 sperm Piwi-RIP-seq data (Fig. 3F), Class I genes were mostly positioned in "Black" Lamin / H1214 and "Blue" Polycomb-associated repressive chromatin annotations (Fig. 3H, Class I). 215 Interestingly, while not enriched in the IP, Class II genes also showed similar chromatin state 216 association (Fig. 3H, Class II). Both of these annotations have been suggested to include 217 enrichments for developmentally naive chromatin compartments and the data are therefore 218 coherent with a mechanism for modulation of early embryo development. No such enrichment was 
219 found for Class III genes (Fig. 3H, Class III). These data are consistent with a mechanism whereby

220 Class I and Class II $s R N A s$ promote targeted, chromatin state specific silencing of offspring gene

221 transcription. Thus, paternal Piwi-bound SRNAs and paternal PIWI-sensitive sRNAs, respectively,

222 are necessary for next-generation repression of Class I \& II genes.

224 The piRNA-pathway is required for paternal inheritance of metabolic state

225 We previously identified similar chromatin-state and metabolic pathway signatures in offspring of 226 a paternal diet-induced model of InterGenerational Metabolic Reprogramming (IGMR) ${ }^{30}$. In that 227 model (depicted in Fig. 4A), a two-day dietary sugar intervention in fathers before mating leads to 228 obesity in adult offspring $(\sim 10 \%$ increase in adult fat content), and in embryos, to transcriptional 229 changes reminiscent of chromatin silencer deficiency. Guided by the similarities we tested for 230 evidence of piRNA-pathway dependency in IGMR. We compared expression of the different 231 Classes identified above (Fig. 3) to datasets from IGMR offspring. Intriguingly, while Class I 232 genes showed no evidence of regulation in IGMR, Class II (piRNA-pathway dosage sensitive) 233 genes showed significant upregulation in offspring of high sugar fed fathers (green; Fig. 4B), 234 including strong enrichment of the most upregulated IGMR genes (upper bulge in the green violin; 235 rank analysis, Fig. 4C). Embryonic transcription of Class II genes is thus sensitive to paternal 236 dietary sugar; from a different point-of-view, diet-triggered intergenerational responses mimic 237 those induced by aub and piwi heterozygosity in the male germline (paternal-aub ${ }^{H e t}$ and -piwi ${ }^{H e t}$ ). 238 Signed rank correlation (Fig. 4D) and RRHO (Fig. S3A) analysis validated these findings genome239 wide. Comparing IGMR and maternal-aub $b^{\text {Het }}$-induced intergenerational responses did not show 240 the same trend (Fig. 4D and S3B). The converse analysis, examining the most up- and down241 regulated IGMR genes, confirmed these findings: Genes most upregulated in high sugar sired 242 embryos showed the same signatures as Class II genes described above: they were upregulated in 243 paternal, but downregulated in maternal $a u b^{\text {Het }}$ crosses (Fig S3C), and sRNAs mapping to these 244 genes were downregulated in $a u b^{\text {Het }}$ mutant sperm and depleted from the Piwi pulldown (Fig S3D). 245 Thus, paternal dietary sugar and piRNA-pathway deficiency trigger comparable intergenerational 246 transcriptional rewiring in offspring. This indicates that IGMR is dependent on the dosage of sperm 247 piRNA-pathway-regulated sRNAs.

248 If these piRNA sensitive genesets were causal with respect to intergenerational reprogramming, 249 we reasoned that piRNA-pathway mutant fathers should fail to trigger an IGMR response. To this 
250 end, we tested whether piRNA-pathway heterozygote fathers ( $p i w i^{H e t}$ and $a u b^{H e t}$ ) were capable of

251 eliciting a full IGMR obesity response using our previously published intergenerational dietary

252 model. We performed the intergenerational diet experiment using WT and piwi ${ }^{H e t}$ and $a u b^{\text {Het }}$

253 fathers. Despite normal fertility and reproductive function in $p i w i^{H e t}$ and $a u b^{H e t}$ fathers, we found

254 that both heterozygote lines failed to elicit increased triglyceride accumulation in the next

255 generation (Fig. 4F). Thus, full piRNA-pathway dosage is necessary for paternal diet-induced

256 intergenerational obesity (IGMR).

258 In summary, we describe a highly dynamic sRNA repertoire during Drosophila spermatogenesis.

259 We find that sperm contains full-length piRNA-pathway proteins, and, using pulldown

260 approaches, prove the existence of Piwi-bound piRNAs in sperm. Heterozygous mutations in

261 piRNA-pathway member proteins lead to changes in sperm sRNAs indicating that mild pathway

262 disruption is sufficient to alter the sperm sRNA load transferred to the zygote and to alter

263 transcription in the next generation early life. Significant correlation between paternal Piwi bound

264 (Class I) and Piwi sensitive (Class II) sRNAs and offspring gene transcription indicates that

265 paternal sRNAs are involved in intergenerational inheritance by targeting sequence-matched genes

266 for silencing in the next generation. Indeed, using paternal sugar triggered IGMR as a test case,

267 we confirm this hypothesis and provide genetic evidence that a fully intact piRNA-pathway is

268 necessary for intergenerational inheritance of paternal metabolic state (Summarized in Fig. 4G). 
Fig. 1:

A
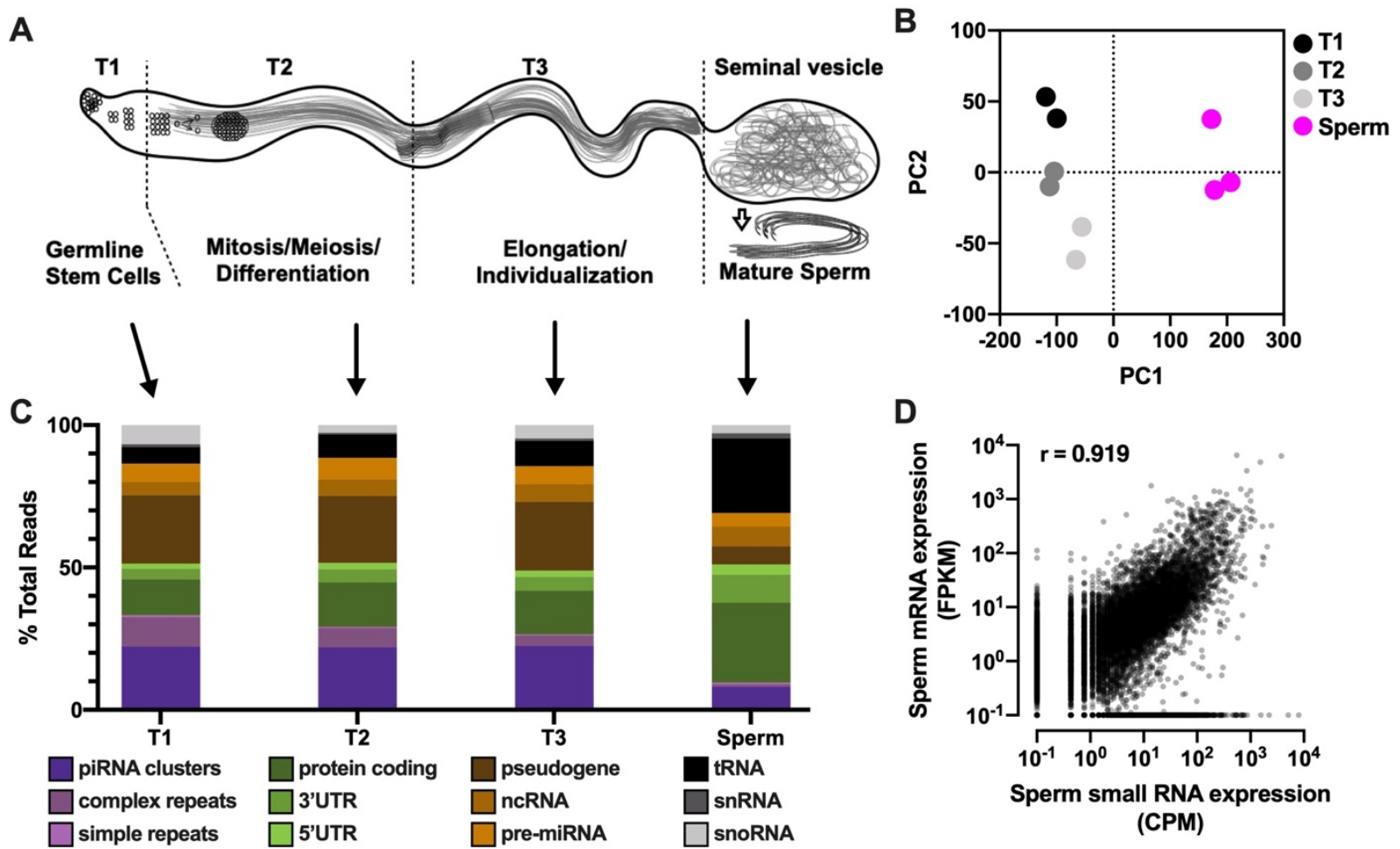

D

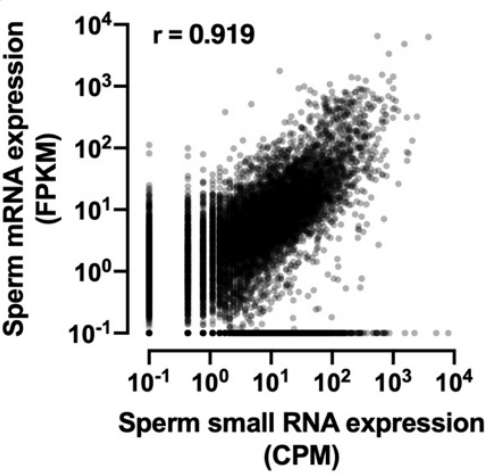

E Drosophila Sperm

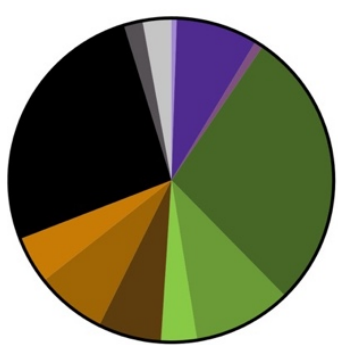

$\mathbf{F}$

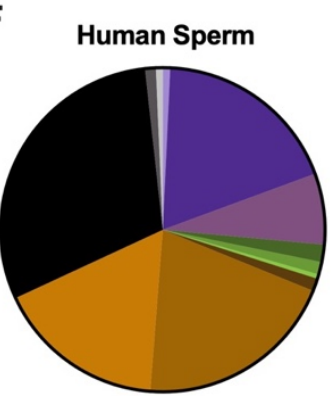

G

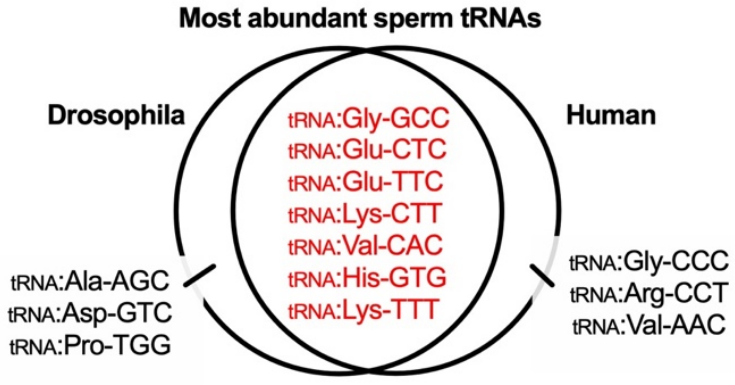

Fig. 1: Dynamic sRNA expression in the male Drosophila germline

A) A schematic of the Drosophila testis indicating segments used for sRNA sequencing; B) PCA plot of sRNA sequencing data; C) \% read distribution across biotypes and across testis segments; biotype color legend applies to panels $\mathrm{C}, \mathrm{E}$ and $\mathrm{F}$; D) correlation of sRNA sequencing results with previously published mRNA sequencing ${ }^{30}$ results from mature sperm and showing Peason's r; E) Drosophila sperm and F) human sperm sRNA biotype distributions; G) 10 most enriched tRNA features in Drosophila and human sperm ${ }^{17}$ sRNA sequencing. 
Fig. 2:

A
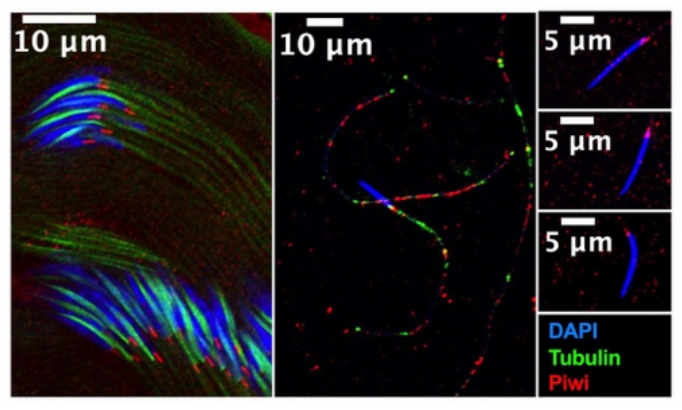

D
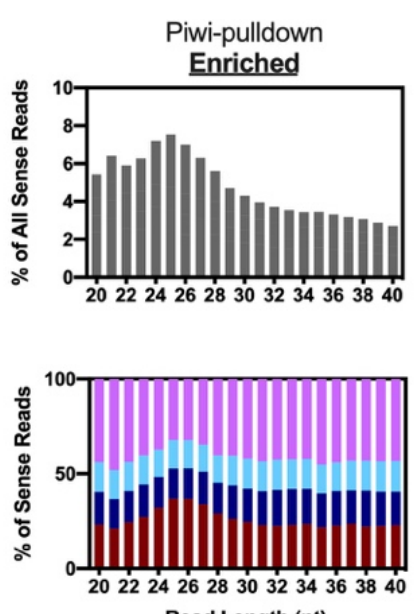

Read Length (nt)

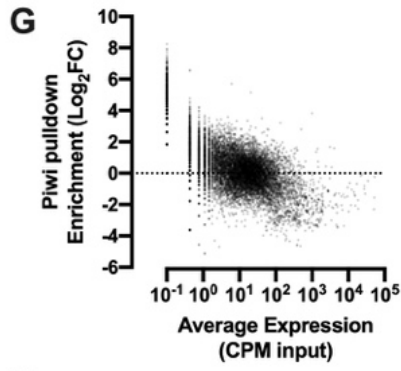

H

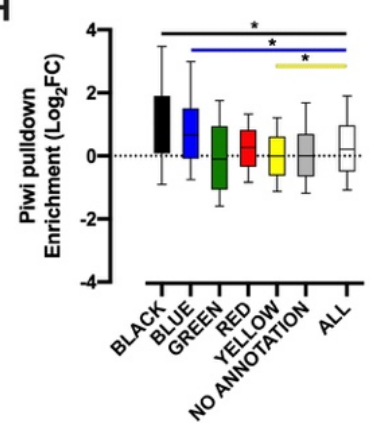

B

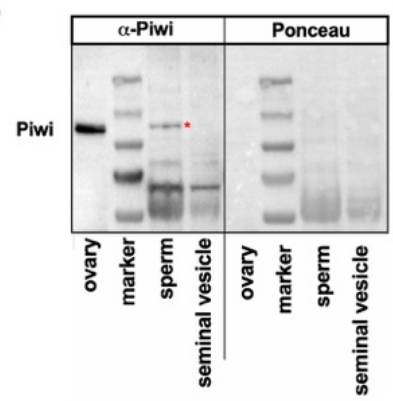

F

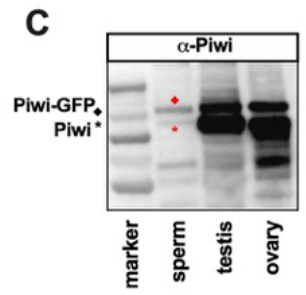

E
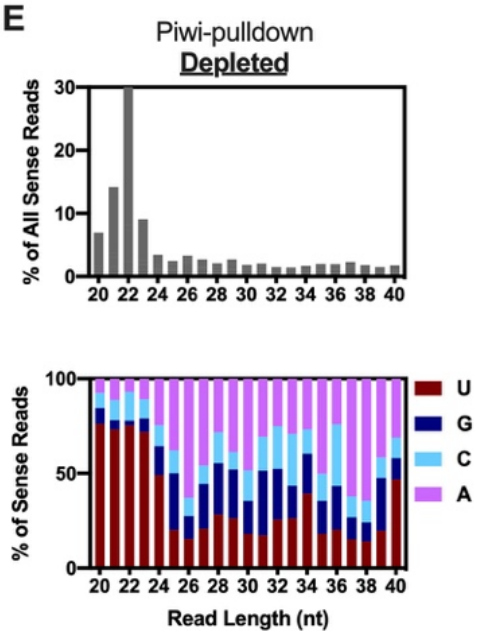

I

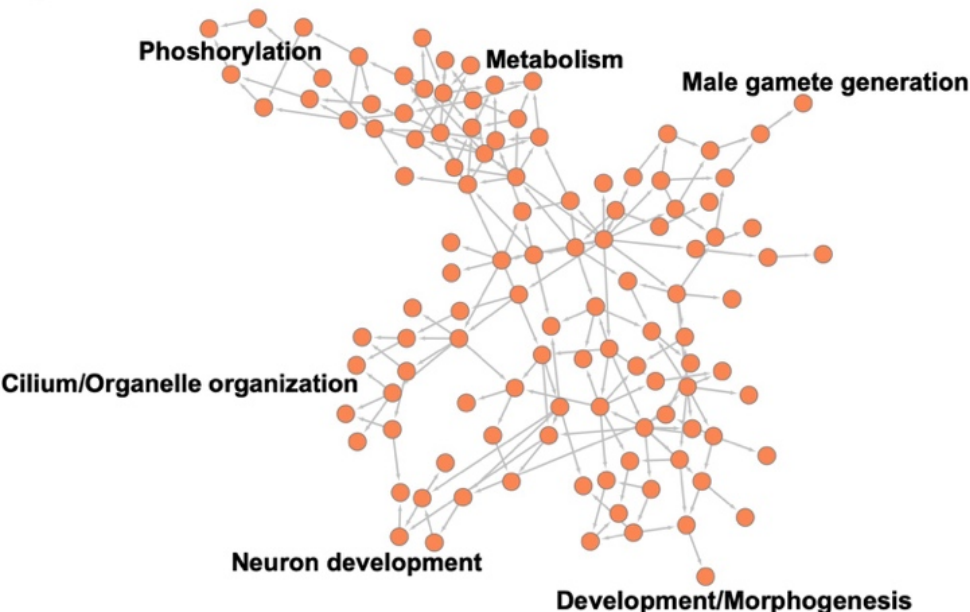

Fig. 2: Presence of Piwi bound RNAs in mature Drosophila sperm A) immunofluorescence staining of sperm with Piwi (ab5207) and tubulin antibodies; B) left panel shows western blot with Piwi antibody (ab5207) for ovary, sperm and empty seminal vesicle, right panel shows Ponceau staining to show protein content per lane. C) sperm, testis and ovaries from GFP-Piwi flies, the upper band shows the GFP fusion protein, lower band shows WT Piwi; D-E) length distribution 
286 (left) and $1^{\text {st }}$ nucleotide bias (right) of sense reads mapping to all features displayed as percentages 287 of all reads between the length of 20-50nt for significantly enriched (D) and for significantly 288 depleted features in Piwi-IP (piwi-antibody ab5207)(E); F) GSEA analysis of sRNA results using 289 custom pathways for the individual biotypes used for annotation; G) average counts per million 290 (CPM) in input samples versus $\log _{2} \mathrm{FC}$ in Piwi pulldown samples $\mathrm{H}$ ) enrichment of reads mapping 291 to features embedded in different chromatin states. I) Pathway overrepresentation analysis 292 (WebGestalt) ${ }^{31}$ of protein coding features significantly enriched in Piwi pulldown. 
Fig.3:
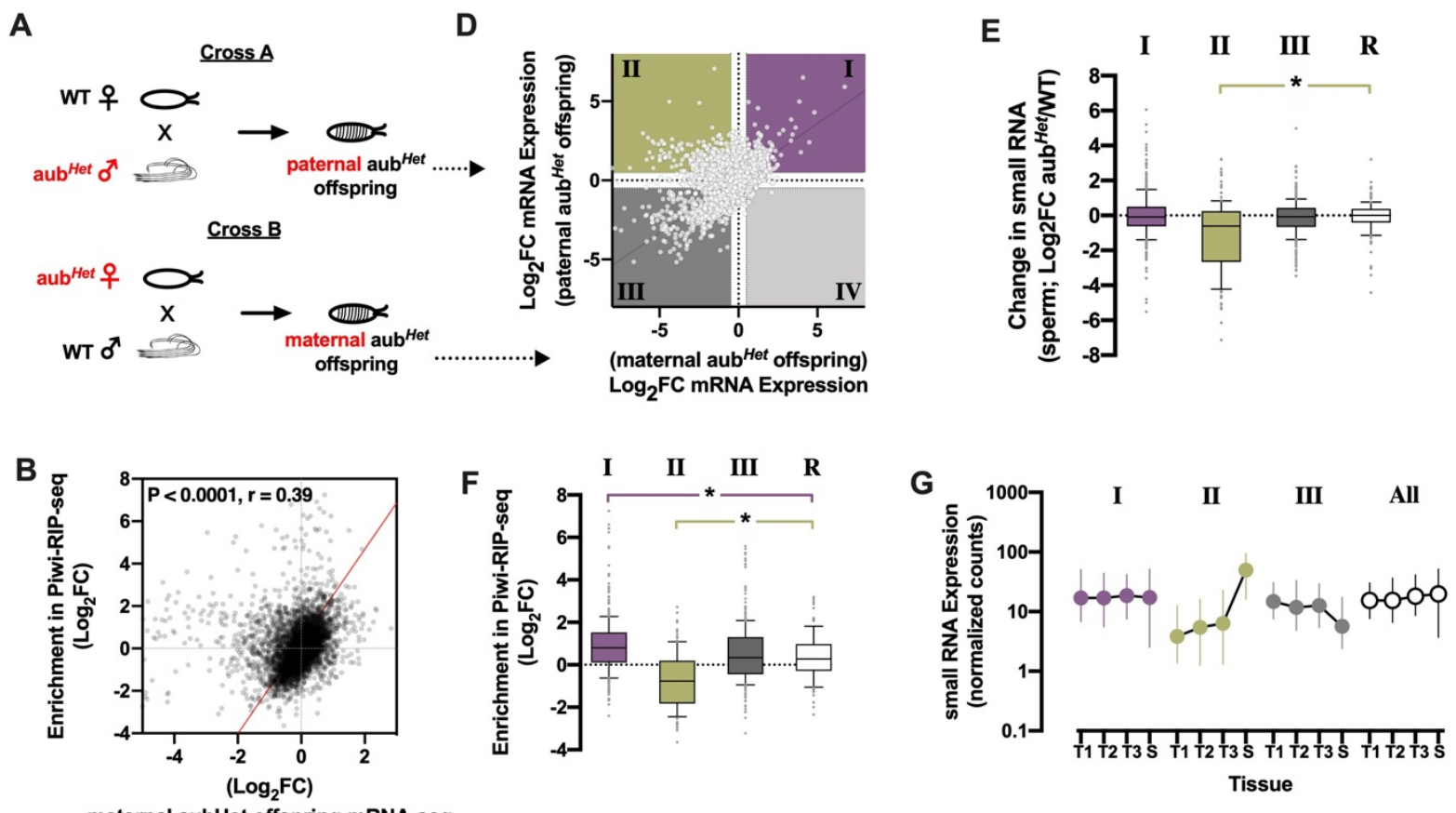

C

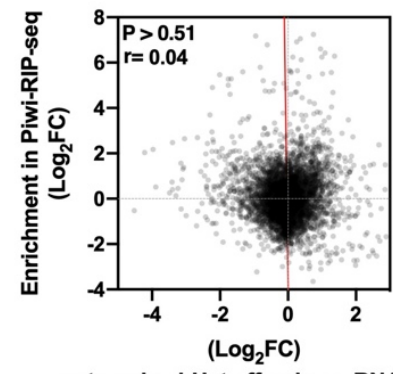

$\mathbf{H}$
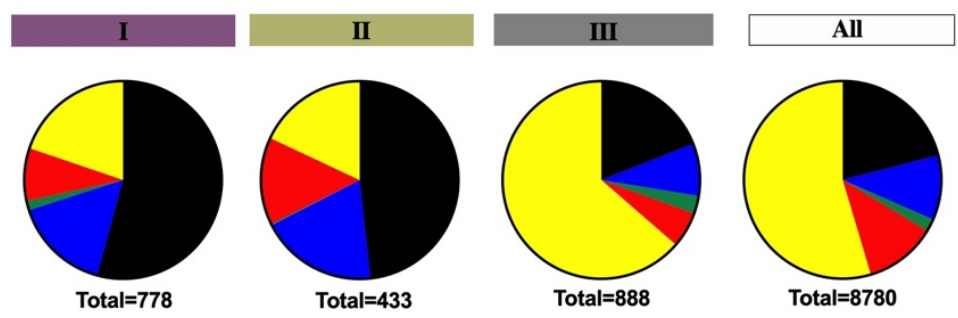

paternal aubHet offspring mRNA-seq

Fig. 3: Paternal piRNAs reprogram offspring

A) crossing scheme; B) comparison of mRNA expression changes induced by paternal vs maternal $a u b^{\text {Het }}$, coloring and numbering is kept consistent throughout manuscript; C) correlation between Piwi pulldown enrichment and mRNA expression changes in maternal aub ${ }^{\text {Het }}$ offspring; D) correlation between Piwi pulldown enrichment and mRNA expression changes in paternal $a u b^{\text {Het }}$ offspring; E ) $\log _{2} \mathrm{FC}$ of sperm sRNAs in $a u b^{\text {Het }}$ vs WT males, each boxplot represents genes present in a different quadrants (Class) in $\mathrm{D}$, random gene set in white F) Enrichment ( $\log _{2}$ FC IP/Input) of sequence matching sRNAs in Piwi pulldown (Piwi-RIP-seq), each boxplot represents sequences matching genes present in a different quadrant (Class/color) from $\mathrm{E}$; $\mathrm{G}$ ) normalized counts in sRNA testis samples (as shown in Fig. 1) mapping to features in different quadrants in D, all genes in white; $\mathrm{H}$ ) chromatin state annotations of Class I-III genes compared to all genes (right). Paternal $a u b^{\text {Het }}$ offspring $\mathrm{n}=5$, WT offspring $\mathrm{n}=5$, maternal $a u b^{\text {Het }}$ offspring $n=2$ with each replicate containing a pool of 20 embryos. 
Fig. 4:

A

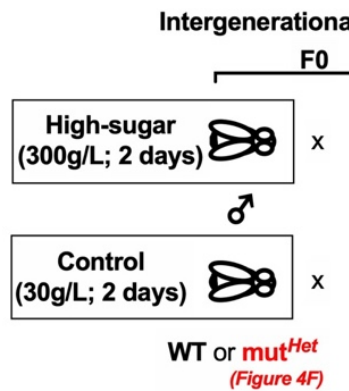

C

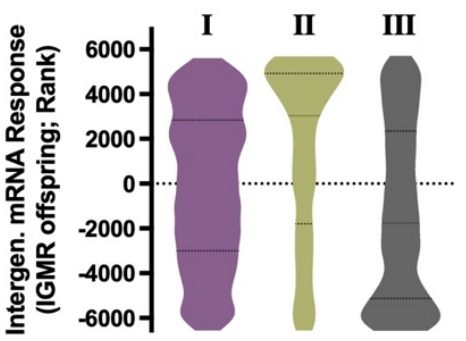

$\mathbf{F}$

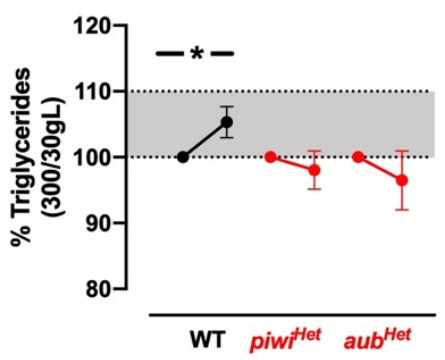

B

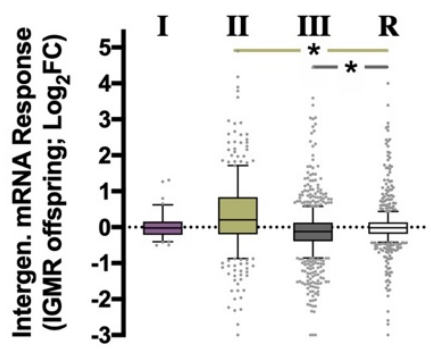

E

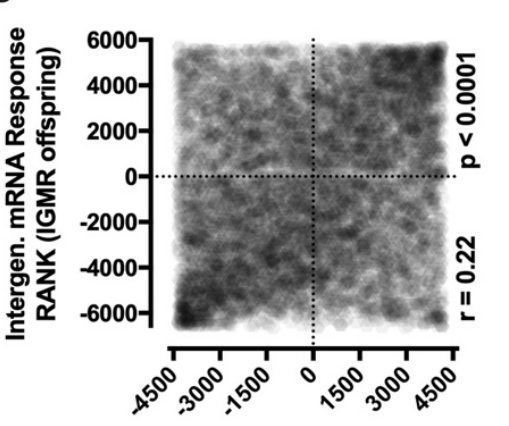

Intergen. mRNA Response RANK (paternal-aub ${ }^{\text {Het }}$ offspring)

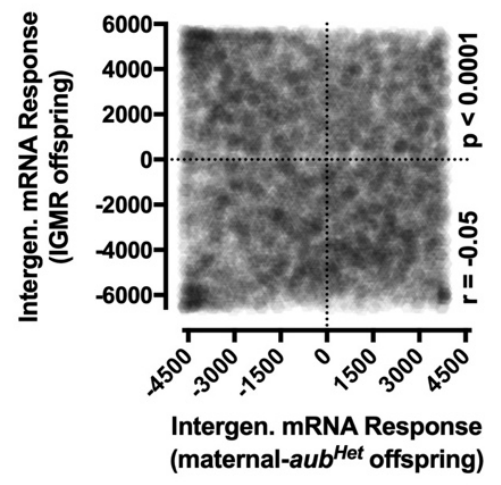

G

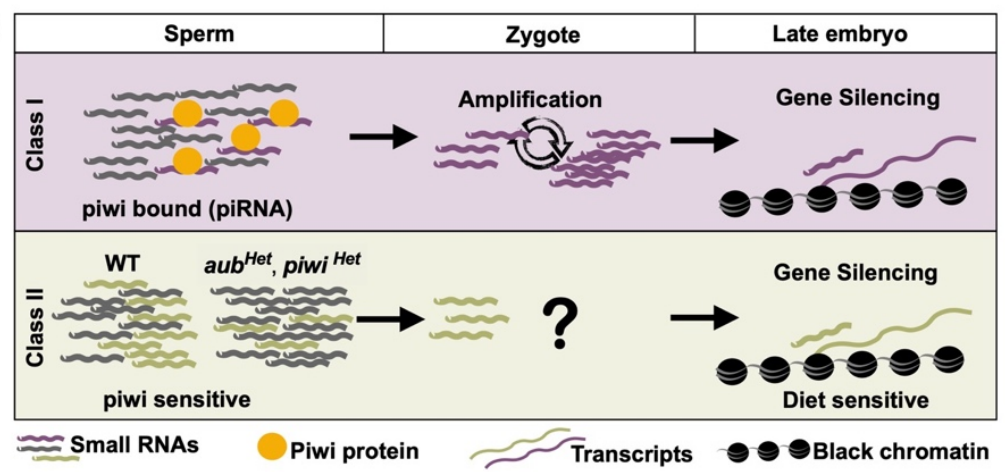

Fig. 4: An intact piRNA-pathway is necessary for the intergenerational inheritance of a physiological phenotype A) crossing scheme for paternal diet-induced intergenerational metabolic reprogramming (IGMR); B) mRNA-seq of IGMR offspring embryos, Log $_{2} \mathrm{FC}$ control vs paternal high-sugar for different Classes as introduced in Fig. $3 \mathrm{~B}$ ( $\mathrm{R}=$ random gene set); $\mathrm{C}$ ) same as $\mathrm{B}$ but rank analysis instead of $\log _{2} \mathrm{FC}$; D) rank analysis comparing mRNA-seq of IGMR offspring embryos to paternal $a u b^{\text {Het }}$ offspring E) rank analysis comparing mRNA-seq of IGMR offspring embryos to maternal $a u b^{\text {Het }}$ offspring, $\mathrm{p}$ value and pearson $\mathrm{r}$ are depicted in $\mathrm{D}$ and $\mathrm{E} ; \mathrm{F}$ ) whole body triglycerides of IGMR offspring as \% of control-sugar matched value using wildtype (WT) or PIWI pathway heterozygous mutant (piwi, aub) fathers $n=12-25$ individual triglyceride assays of groups of 5 flies from at least 3 independent experiments; G) proposed model and summary for the data presented 
Fig. S1:

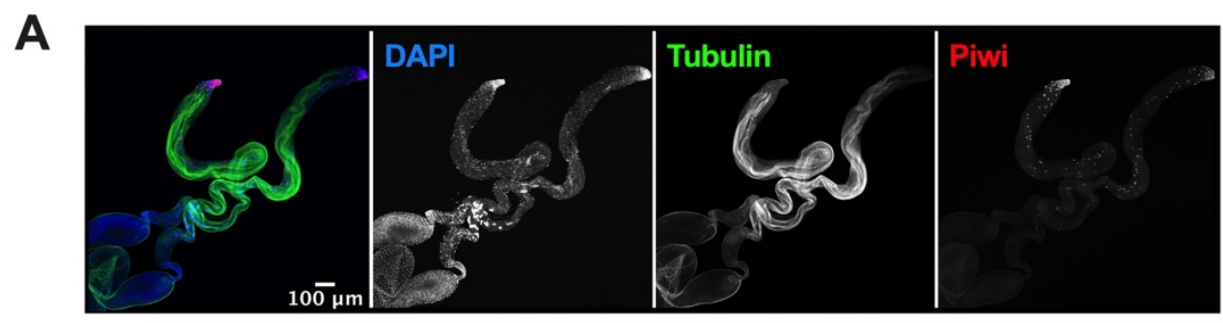

B

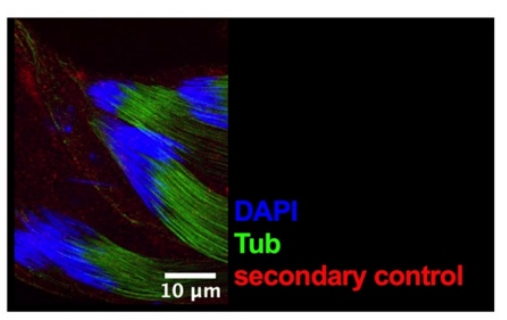

C

\begin{tabular}{|l|r|r|r|}
\hline & Piwi & aub & AGO3 \\
\hline Targeted Peptides & 21 & 10 & 10 \\
\hline Identified Peptides & 21 & 9 & 10 \\
\hline
\end{tabular}

D All genes present

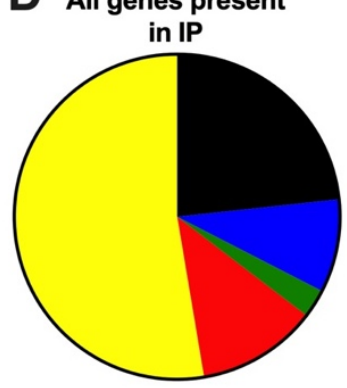

Total $=\mathbf{5 0 7 7}$

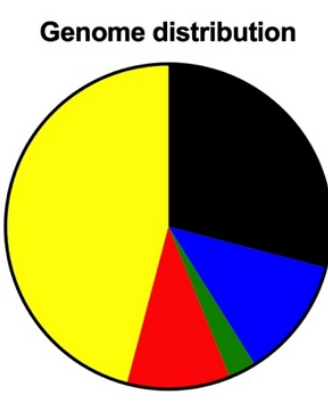

Total=14671
Significantly enriched in IP

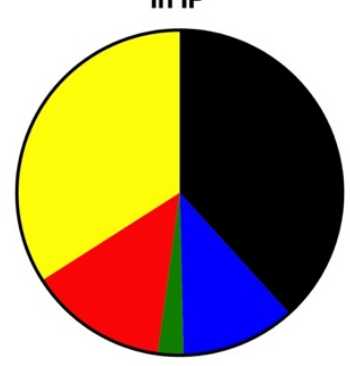

Total $=943$
Significantly depleted from IP

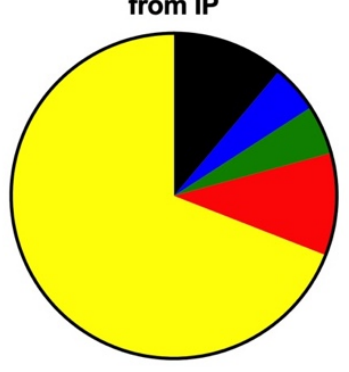

Total $=530$

Fig. S1: Presence of Piwi bound RNAs in mature sperm A) immunofluorescence staining of testis with Piwi (sc-390946) and tubulin antibodies; B) secondary antibody control staining for Piwi (ab5207) staining in Fig. 2A; C) results of targeted mass spectrometry experiment identifying peptides in isolated sperm; D) pie chart representation of chromatin state distribution of reads mapping to features embedded in different chromatin states. 


\section{Fig.S2:}

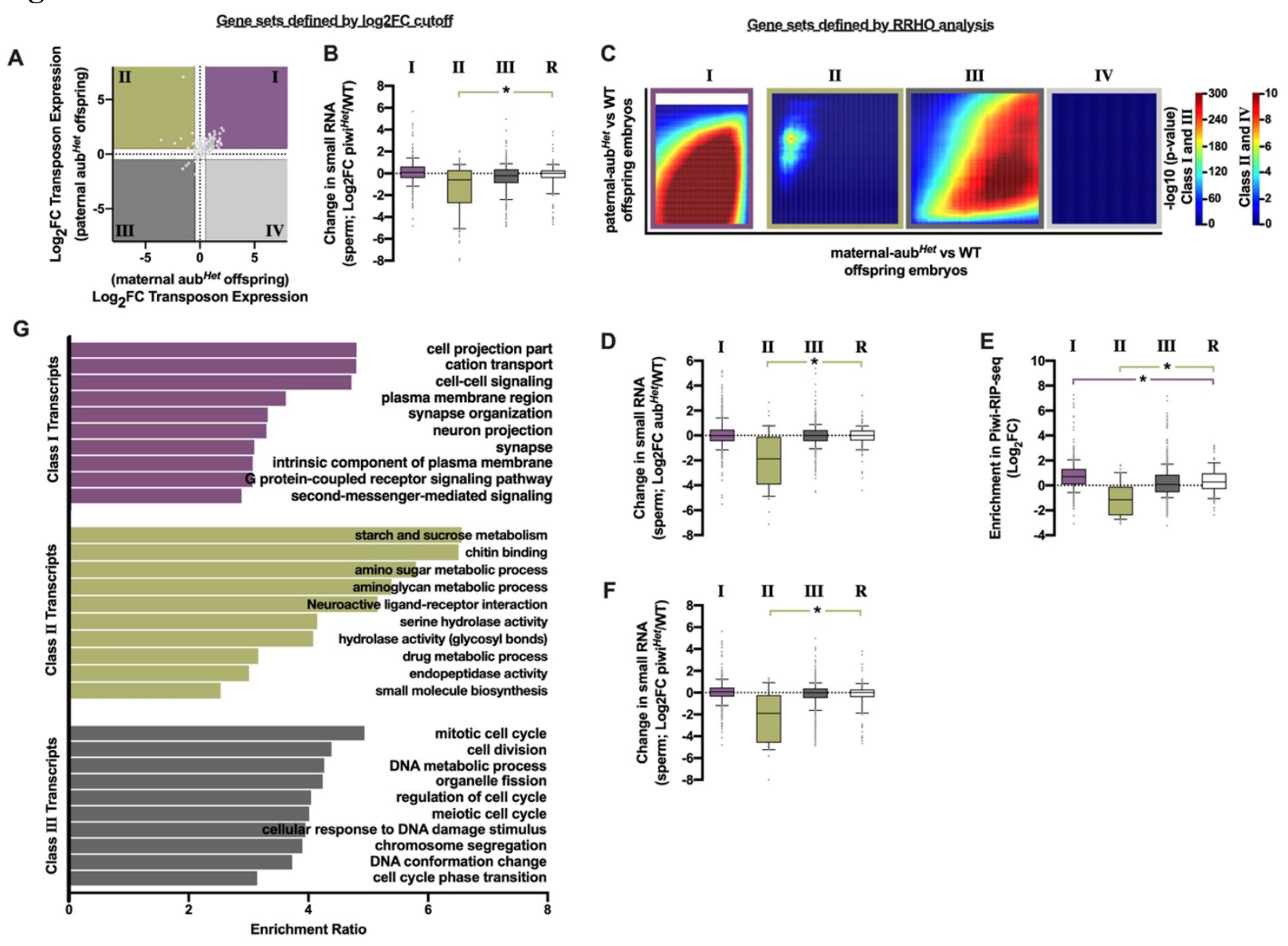

\section{Fig. S2: Paternal piRNAs reprogram offspring}

A) comparison of transposon expression changes induced by paternal and maternal aub ${ }^{\text {Het; }}$ B) $\log _{2} \mathrm{FC}$ of sperm sRNAs piwi ${ }^{\mathrm{Het}} / \mathrm{WT}$, each boxplot represents genes present in a different quadrants (Class) from Fig. 3B, random gene set in white; C) Rank Rank Hypergeometric test of expression changes induced by paternal and maternal $a u b^{\text {Het }}$ confirms three Classes, different scales were applied to Class I/III and Class II/IV as depicted by the scale on the right; D) $\log _{2} \mathrm{FC}$ of sperm sRNAs $a u b^{H e t} / \mathrm{WT}$, each boxplot represents genes present in a different quadrants from RRHO analysis in $\mathrm{C}$, random gene set in white; E) Enrichment ( $\log _{2} \mathrm{FC} \mathrm{IP/Input)} \mathrm{in} \mathrm{piwi} \mathrm{pulldown}$ experiment, each boxplot represents genes present in a different quadrants from RRHO analysis in $\mathrm{C}$, random gene set in white; F) $\log _{2} \mathrm{FC}$ of sperm sRNAs $\mathrm{piwi}^{\mathrm{Het}} / \mathrm{WT}$, each boxplot represents genes present in a different quadrants from RRHO analysis in C, random gene set in white; G) over representation analysis of different Classes depicted in Fig. 3B using WebGestalt on gene ontology terms included in Biological Processes noRedundant 
Fig. S3:

A

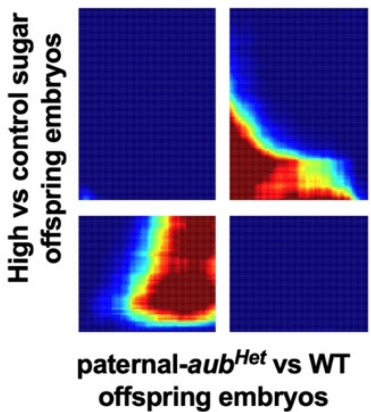

C

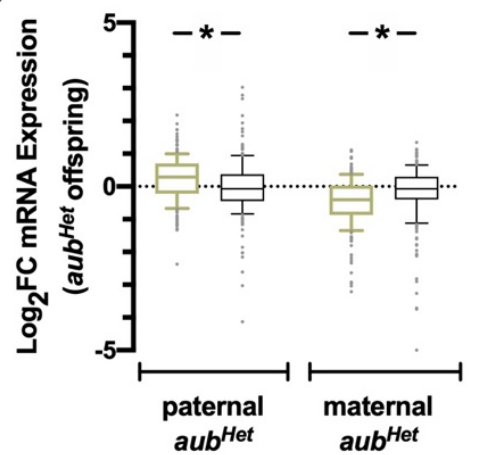

B
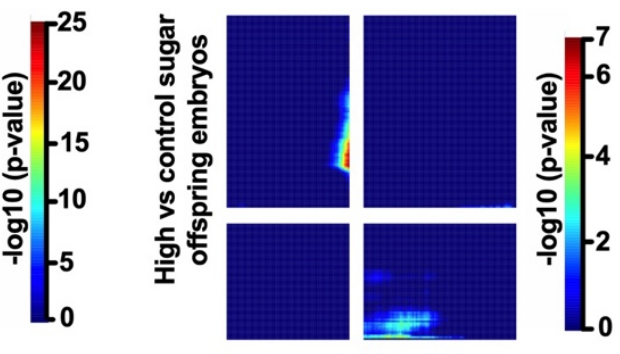

maternal-aub ${ }^{\text {Het }}$ vs WT offspring embryos

D

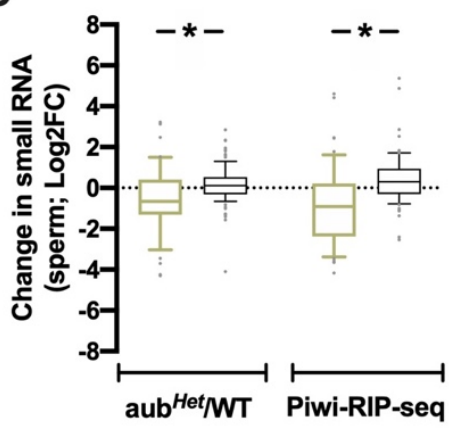

Fig. S3: An intact piRNA-pathway is necessary for the intergenerational inheritance of a physiological phenotype. Rank Rank Hypergeometric test of expression changes in embryos induced by paternal diet intervention and A) paternal $a u b^{\text {Het }} \mathrm{B}$ ) maternal $a u b^{\text {Het }}$; C) $\log _{2} \mathrm{FC}$ mRNA expression changes of the 300 most upregulated genes in IGMR offspring in paternal (green, left) and maternal (green, right) $a u b^{\text {Het }}$ offspring; D) $\log _{2} \mathrm{FC}$ changes of sperm sRNA abundance in $a u b^{\text {Het }}$ sperm (green, left) and IP/Input (green, right), for those sRNAs mapping to the 300 most upregulated genes in IGMR offspring random gene set in black. 


\section{References and Notes:}

3601 Czech, B. et al. piRNA-Guided Genome Defense: From Biogenesis to Silencing. Annu Rev Genet 52, 131-157, doi:10.1146/annurev-genet-120417-031441 (2018). Ozata, D. M., Gainetdinov, I., Zoch, A., O'Carroll, D. \& Zamore, P. D. PIWl-interacting RNAs: small RNAs with big functions. Nat Rev Genet 20, 89-108, doi:10.1038/s41576018-0073-3 (2019). vanguard of genome defence. Nat Rev Mol Cell Biol 12, 246-258, doi:10.1038/nrm3089 Biogenesis, Initiated and Directed by PIWI Proteins, Explains piRNA Production in Most Animals. Mol Cell 71, 775-790 e775, doi:10.1016/j.molcel.2018.08.007 (2018). novo DNA methylation in mice. Mol Cell 31, 785-799, doi:10.1016/j.molcel.2008.09.003 (2008).

6 Robine, N. et al. A broadly conserved pathway generates 3'UTR-directed primary piRNAs. Curr Biol 19, 2066-2076, doi:10.1016/j.cub.2009.11.064 (2009).

7 Mohn, F., Handler, D. \& Brennecke, J. Noncoding RNA. piRNA-guided slicing specifies transcripts for Zucchini-dependent, phased piRNA biogenesis. Science 348, 812-817, doi:10.1126/science.aaa1039 (2015).

$8 \mathrm{Ha}, \mathrm{H}$. et al. A comprehensive analysis of piRNAs from adult human testis and their relationship with genes and mobile elements. BMC Genomics 15, 545, doi:10.1186/1471-2164-15-545 (2014).

9 Yamtich, J., Heo, S. J., Dhahbi, J., Martin, D. I. \& Boffelli, D. piRNA-like small RNAs mark extended 3'UTRs present in germ and somatic cells. BMC Genomics 16, 462, doi:10.1186/s12864-015-1662-6 (2015).

10 Saito, K. et al. A regulatory circuit for piwi by the large Maf gene traffic jam in Drosophila. Nature 461, 1296-1299, doi:10.1038/nature08501 (2009).

11 Aravin, A. A. et al. Double-stranded RNA-mediated silencing of genomic tandem repeats and transposable elements in the D. melanogaster germline. Curr Biol 11, 1017-1027, doi:10.1016/s0960-9822(01)00299-8 (2001).

12 Huang, X., Fejes Toth, K. \& Aravin, A. A. piRNA Biogenesis in Drosophila melanogaster. Trends Genet 33, 882-894, doi:10.1016/j.tig.2017.09.002 (2017).

13 Ashe, A. et al. piRNAs can trigger a multigenerational epigenetic memory in the germline of C. elegans. Cell 150, 88-99, doi:10.1016/j.cell.2012.06.018 (2012).

14 Malone, C. D., Lehmann, R. \& Teixeira, F. K. The cellular basis of hybrid dysgenesis and Stellate regulation in Drosophila. Curr Opin Genet Dev 34, 88-94, doi:10.1016/j.gde.2015.09.003 (2015).

15 Papagiannouli, F. \& Lohmann, I. Shaping the niche: lessons from the Drosophila testis and other model systems. Biotechnol J 7, 723-736, doi:10.1002/biot.201100352 (2012).

16 Sharma, U. et al. Small RNAs Are Trafficked from the Epididymis to Developing Mammalian Sperm. Dev Cell 46, 481-494 e486, doi:10.1016/j.devcel.2018.06.023 (2018).

17 Natt, D. et al. Human sperm displays rapid responses to diet. PLoS Biol 17, e3000559, doi:10.1371/journal.pbio.3000559 (2019).

Brennecke, J. et al. Discrete small RNA-generating loci as master regulators of transposon activity in Drosophila. Cell 128, 1089-1103, doi:10.1016/j.cell.2007.01.043 (2007). 442, 203-207, doi:10.1038/nature04916 (2006). 
40920 Grivna, S. T., Beyret, E., Wang, Z. \& Lin, H. A novel class of small RNAs in mouse spermatogenic cells. Genes Dev 20, 1709-1714, doi:10.1101/gad.1434406 (2006). Lau, N. C. et al. Characterization of the piRNA complex from rat testes. Science 313, 363-367, doi:10.1126/science.1130164 (2006). of small RNAs binds mammalian Piwi proteins. Nature 442, 199-202, doi:10.1038/nature04917 (2006).

23 Gonzalez, J., Qi, H., Liu, N. \& Lin, H. Piwi Is a Key Regulator of Both Somatic and Germline Stem Cells in the Drosophila Testis. Cell Rep 12, 150-161, doi:10.1016/j.celrep.2015.06.004 (2015).

24 Hutcheon, K. et al. Analysis of the small non-protein-coding RNA profile of mouse spermatozoa reveals specific enrichment of piRNAs within mature spermatozoa. RNA Biol 14, 1776-1790, doi:10.1080/15476286.2017.1356569 (2017).

25 Filion, G. J. et al. Systematic protein location mapping reveals five principal chromatin types in Drosophila cells. Cell 143, 212-224, doi:10.1016/j.cell.2010.09.009 (2010).

26 Han, B. W., Wang, W., Li, C., Weng, Z. \& Zamore, P. D. Noncoding RNA. piRNA-guided transposon cleavage initiates Zucchini-dependent, phased piRNA production. Science 348, 817-821, doi:10.1126/science.aaa1264 (2015).

27 Senti, K. A., Jurczak, D., Sachidanandam, R. \& Brennecke, J. piRNA-guided slicing of transposon transcripts enforces their transcriptional silencing via specifying the nuclear piRNA repertoire. Genes Dev 29, 1747-1762, doi:10.1101/gad.267252.115 (2015).

$28 \mathrm{Gu}, \mathrm{T}$. \& Elgin, S. C. Maternal depletion of Piwi, a component of the RNAi system, impacts heterochromatin formation in Drosophila. PLoS Genet 9, e1003780, doi:10.1371/journal.pgen.1003780 (2013).

29 Cahill, K. M., Huo, Z., Tseng, G. C., Logan, R. W. \& Seney, M. L. Improved identification of concordant and discordant gene expression signatures using an updated rank-rank hypergeometric overlap approach. Sci Rep 8, 9588, doi:10.1038/s41598-018-27903-2 (2018).

30 Ost, A. et al. Paternal diet defines offspring chromatin state and intergenerational obesity. Cell 159, 1352-1364, doi:10.1016/j.cell.2014.11.005 (2014).

31 Liao, Y., Wang, J., Jaehnig, E. J., Shi, Z. \& Zhang, B. WebGestalt 2019: gene set analysis toolkit with revamped Uls and APIs. Nucleic Acids Res 47, W199-W205, doi:10.1093/nar/gkz401 (2019).

32 Piper, M. D. et al. A holidic medium for Drosophila melanogaster. Nat Methods 11, 100105, doi:10.1038/nmeth.2731 (2014).

33 Raich, N., Contremoulins, V. \& Karess, R. E. Immunostaining of Whole-Mount Drosophila Testes for 3D Confocal Analysis of Large Spermatocytes. J Vis Exp, doi:10.3791/61061 (2020).

34 Hayashi, R. et al. Genetic and mechanistic diversity of piRNA 3'-end formation. Nature 539, 588-592, doi:10.1038/nature20162 (2016).

35 Martin, M. Cutadapt removes adapter sequences from high-throughput sequencing reads. EMBnet.journal 17, 10-12, doi:DOI: http://dx.doi.org/10.14806/ej.17.1.200 (2011).

36 Langmead, B. Aligning short sequencing reads with Bowtie. Curr Protoc Bioinformatics Chapter 11, Unit 11 17, doi:10.1002/0471250953.bi1107s32 (2010).

37 Liao, Y., Smyth, G. K. \& Shi, W. featureCounts: an efficient general purpose program for assigning sequence reads to genomic features. Bioinformatics 30, 923-930, doi:10.1093/bioinformatics/btt656 (2014).

38 Robinson, M. D., McCarthy, D. J. \& Smyth, G. K. edgeR: a Bioconductor package for differential expression analysis of digital gene expression data. Bioinformatics 26, 139140, doi:10.1093/bioinformatics/btp616 (2010). 
39 Yu, G., Wang, L. G., Han, Y. \& He, Q. Y. clusterProfiler: an R package for comparing biological themes among gene clusters. OMICS 16, 284-287, doi:10.1089/omi.2011.0118 (2012).

\section{Author Contributions:}

A.L., A.Ö. and J.A.P. conceived, designed and supervised the study. A.L. and J.A.P wrote the manuscript with feedback from all authors. A.L., L.E., A.G.M, O.L., M.S, U.K., M.I., L.Ö., M.G.L.R, E.B., performed fly work and dissections. U.K. perform library preparation for small RNA sequencing. L.E. and M.I. optimized and performed piwi RIP-seq. A.L. performed immunostaining. A.G.M. performed western blot analysis. T.R. prepared samples for mass spec. E.B. performed embryo mRNA sequencing. E.C., I.B., D.N., A.L., D.P. performed bioinformatic analysis. A.L. and T.V. supervised bioinformatic analysis.

\section{Funding sources:}

474 This work was supported by the Knut och Alice Wallenbergs Stiftelse [2015.0165]; Ragnar

475 Söderbergs stiftelse; Vetenskapsrådet [201503141]; European Research Council

476 [ERC-StG-281641]. 


\section{Supplementary Materials:}

478 Materials and Methods

$479 \quad$ Fly Food

480 Standard food: Agar $12 \mathrm{~g} / \mathrm{l}$, yeast $18 \mathrm{~g} / \mathrm{l}$, soy flour $10 \mathrm{~g} / 1$, yellow cornmeal $80 \mathrm{~g} / 1$, molasses $22 \mathrm{~g} / 1$, malt extract $80 \mathrm{~g} / \mathrm{l}$, Nipagin $24 \mathrm{~g} / \mathrm{l}$, propionic acid $6,25 \mathrm{ml} / \mathrm{l}$. Holidic diet was prepared according to ${ }^{32}$.

Fly stocks

w1118: used as WT stock $\mathrm{aub}^{\mathrm{Qc42}}$ - BDSC \#4968: used for genetic crosses piwi $^{1}$ - BDSC \#43637: used for genetic crosses T(2;3)TSTL, CyO: TM6B, Tb[1] - BDSC \#42713: double balancer used for back-crossing

Fly Husbandry

Fly stocks were maintained on standard-diet at $25^{\circ} \mathrm{C}$ on a 2 -week generation cycle. To ensure a common parental larva density and epigenetic background, 0-4 days old flies were manually sorted to 15 males and 15 females per vial (or $45+45$ in bottles) and allowed to lay eggs for 4 days. In practice, flies were crossed on Thursday, flipped out on Monday and virgins collected the following Monday. On Thursday flies were crossed again fulfilling a 2-week generation cycle.

Generation of flies for genetic crosses

498 To equalize the genetic background between mutant and WT strains we crossed the mutant lines over double balancer lines so chromosomes can be followed using a visible marker. These flies were then crossed to the WT strain and all chromosomes were replaced with WT chromosomes except the one carrying the mutation and the respective balancer. Flies from the above cross were crossed with WT and the resulting offspring without the balancer chromosome, was used for the for the paternal and maternal mut ${ }^{H e t}$ crosses.

Testis, sperm sack and ovary dissection

The reproductive tract of separated male and female flies was dissected in a drop of TC-100 insect medium (Sigma) and connective tissue and other contaminating tissues were removed. Ovaries were transferred to a tube containing insect medium. Testes with attached seminal vesicles (sperm sacks) were transferred to a fresh drop of insect medium, where the sperm sack was separated from the rest of the testis. Testes were further separated into three parts and transferred to different tubes containing insect medium. Mature sperm was removed from the sperm sacks by puncturing them and spooling the sperm onto clean forceps and transferred to a tube containing insect medium. After a maximum of 30 min of dissection, collected tissues were snap frozen in liquid nitrogen. When enough samples were collected the tissues were pelleted, the supernatant was discarded, and the sperm pellet was re-suspended in $250 \mu$ l of Trizol (Invitrogen).

\section{Collection of staged D. melanogaster embryos}

Virgin female flies were kept in egg-laying cages with apple-juice plates supplied with fresh yeast paste for three days. Female virgin flies were crossed to male virgin flies in egg-laying cages at 25 ${ }^{\circ} \mathrm{C}$ in the morning and apple juice plates were changed approximately every $30 \mathrm{~min}$ until $2 \mathrm{pm}$. Subsequent apple juice plates were used for collection of embryos. To obtain stage 17 embryos pates were left on the cage for $2 \mathrm{~h}$ and incubated for $16 \mathrm{~h}$ at $25^{\circ} \mathrm{C}$. Embryos on apple juice plates 
were dechorionated using 50\% PBS-TX (PBS-T containing $0.3 \%$ Triton X-100) and $50 \%$ bleach for $\sim 2$ min. Dechorionated and detached embryos were collected and rinsed under a stream of water. Embryos were then staged according to their morphology to maximize homogeneity in the sample and immediately transferred to $250 \mu 1$ of Trizol solution for RNA isolation.

\section{RNA isolation for sRNA-seq}

Samples prepared in Linkoping: Frozen samples were homogenized in Qiazol with $0.15 \mathrm{~g} 0.2 \mathrm{~mm}$ steel beads, Tissue Lyser 2 min 40 osc. RNA extraction was done using miRNeasy Micro kit (Qiagen, Venlo, the Netherlands) and performed according to the manufacturer instructions, RNA was eluted in $14 \mu \mathrm{l}$ of water and stored at $-70{ }^{\circ} \mathrm{C}$ until library preparation.

Samples prepared in Freiburg: Dried RNA were shipped on dry ice and kept in $-70{ }^{\circ} \mathrm{C}$. Dried and washed once with $80 \%$ ethanol and dried, dissolved in 20 ul water, extraction was done using miRNeasy Micro kit (Qiagen, Venlo, the Netherlands) and performed according to the manufacturer instructions. RNA was eluted in $14 \mu \mathrm{l}$ of water and stored at $-70{ }^{\circ} \mathrm{C}$ until library preparation. Bioanalyzer confirmed the quality of the RNA.

\section{Library preparation for sRNA-seq}

Library preparation was done with NEBNext Small RNA Library Prep Set for Illumina (New England Biolabs, Ipswich, MA) according to the manufacturer instructions with the following minor customizations. All testicle samples, but not sperm samples, were downscaled to half volume, using $3 \mu \mathrm{l}$ of input RNA instead of $6 \mathrm{ul}$ as recommended. In all steps the primers in the kit were diluted 1:3 prior to use. 2S rRNA was blocked by adding anti-sense oligos ( $5^{\prime}$-TAC AAC CCT CAA CCA TAT GTA GTC CAA GCA372 SpcC3 3'; $10 \mu \mathrm{M}$ ) and set to hybridized in the same step as NEBNext SR RT primer (pink). Amplification was made during 16 cycles and amplified libraries were cleaned using Agencourt AMPure XP (Beckman Coulter, Brea, CA) and size selected for 130 to $165 \mathrm{nt}$ fragments on a pre-casted $6 \%$ polyacrylamide Novex TBE gel (Invitrogen, Waltham, MA). Gel extraction was done using Gel breaker tubes (IST Engineering, Milpitas, CA) in the buffer provided in the NEBNext kit. Disintegrated gels were incubated at 37 ${ }^{\circ} \mathrm{C}$ for 1 hour on a shaker, quickly frozen for 15 minutes at $-80^{\circ} \mathrm{C}$, followed by another incubation for 1 hour. Any remaining gel debris was removed by Spin-X $0.45 \mu \mathrm{m}$ centrifuge tubes (Corning Inc., Corning, NY) as recommended by the NEBnext protocol. The libraries were precipitated overnight at $-80{ }^{\circ} \mathrm{C}$ by adding $1 \mu \mathrm{l}$ of GlycoBlue (Invitrogen) as co-precipitant, 0.1 times the volume of Acetate $3 \mathrm{M}$ (pH 5.5), and 3 times the volume of $100 \%$ ethanol. Library concentrations were estimated using QuantiFluor ONE ds DNAsystem on a Quantus fluorometer (Promega, Madison, WI). Pooled libraries were sequenced on NextSeq 500 with NextSeq 500/550 High Output Kit version 2, 75 cycles (Illumina, San Diego, CA). All pooled libraries passed Illumina's default quality control.

\section{Immunostaining}

Immunostaining of whole mount testis and dissected sperm was carried out according to ${ }^{33}$ in short: testes were dissected in insect medium (Sigma, TC-100) and all incubation and washing steps were carried out using home-made baskets in 96 well plates on an orbital shaker. Fixation was carried out in 4\% formaldehyde (methanol-free) in PBST (PBS and 0.2\% Triton X-100) for 10 min at room temperature. After three 5 min washing steps in PBST testes were permeabilized twice in $0.3 \%$ sodium-deoxycholate in PSTX for $30 \mathrm{~min}$ at room temperature. This was followed by three 
5 min washing steps in PBSTX and 1h of blocking with 5\% BSA in PBSTX at room temperature. Primary antibody incubation (anti-Piwi antibody ab5207 - 1:500 dilution; anti-acetylated $\alpha$-tubulin (Lys40) antibody - 1:500, anti-Piwi antibody sc-390946 - 1:500 dilution) was carried out in PBSTX $+3 \%$ BSA at $4{ }^{\circ} \mathrm{C}$ overnight followed by one $20 \mathrm{~min}$ wash with $300 \mathrm{mM} \mathrm{NaCl}$ in PBSTX and three $5 \mathrm{~min}$ washes with PBSTX at room temperature. Secondary anti-Mouse Alexa Fluor 488 (1:1000; Molecular Probes) and anti-rabbit Alexa Fluor 555 (1:1000; Molecular Probes), incubation lasted for $6 \mathrm{~h}$ in PBSTX $+3 \% \mathrm{BSA}$ at $4{ }^{\circ} \mathrm{C}$ samples were washed with $300 \mathrm{mM} \mathrm{NaCl}$ in PBSTX for $20 \mathrm{~min}$ at room temperature, all steps were repeated for the second primary and secondary antibodies and samples were mounted with Vectashield with DAPI (Vector Labs). Confocal images were taken with a Zeiss LSM510 confocal scanning microscope with a CApochromat $\times 63,1.4$ NA oil immersion objective, using the diode $405 \mathrm{~nm}$, the argon $488 \mathrm{~nm}$, the helium-neon $543 \mathrm{~nm}$ laser for excitation of DAPI, Alexa Fluor ${ }^{\circledR}-488,-555$, respectively.

Triglyceride Determination

Groups of five flies (7-12 days old males) were crushed thoroughly in $100 \mu$ l RIPA buffer, sonicated and the homogenates were used for 96-well based colorimetric determination of triglycerides (GPO Trinder, Sigma). Before absorbance measurement, plates were centrifuged, and supernatants transferred to a new plate.

Mass Spectrometry (MS/MS) analysis

Sperm sack and pure sperm samples were analyzed by nanoLC-MS. Cells were lysed with lysis Buffer (4\% SDS, 100mM DTT,50 mM Tris-HCl buffer $\mathrm{pH} 7.5$, plus protease inhibitors (Roche)). Then the extract was heated to $90^{\circ} \mathrm{C}$ for $3 \mathrm{~min}$ followed by residual chromatin shearing in Bioruptor. Before MS-MS analysis samples were analyzed by SDS-PAGE followed by silver staining (Thermofisher).

\section{Western blotting}

For protein extraction tissue were lysate adding $50 \mathrm{ml}$ of Laemmli sample buffer $2 \mathrm{x}$, heated at $95^{\circ} \mathrm{C}$ for $2 \mathrm{~min}$ and sheared in Biorupter (30 sec/hard). Protein lysates were loaded on NUPAGE 4-12\% precast gel (Life Technologies) in NUPAGE 1xMOPS buffer (Invitrogen). PageRuler Plus Prestain Protein Ladder (Thermo Scientific) was used to indicate protein size. Proteins were subsequently transferred to PVDF membranes. Before blocking, membranes were stained with Ponceau solution (Sigma) and images were recorded. Membranes were blocked in phosphate buffered saline plus 0,05\% Tween 20 (PBST) and 5\% BSA for $1 \mathrm{~h}$ at room temperature. Membranes were incubated with primary Anti-Piwi antibody (ab5207) at 1:500 dilution overnight at $4{ }^{\circ} \mathrm{C}$, washed in PBST, and incubated with horseradish peroxidase (HRP) coupled secondary antibodies (Anti-rabbit IgG, HRP-linked Antibody \#7074) in the washing buffer with 1\% skimmed milk in PBST for $1 \mathrm{~h}$ at room temperature. Membranes were developed using SuperSignal ${ }^{\mathrm{TM}}$ West Femto Maximum Sensitivity Substrate (Thermofisher).

611

Ribonucleoprotein Immunoprecipitation (RIP)

611

All steps were performed on ice or at $4{ }^{\circ} \mathrm{C}$ unless indicated otherwise. Samples with magnetic

612 beads were placed on magnetic rack for $30 \mathrm{sec}$ to ensure beads were pelleted. Dissected tissues 614 inhibitor. Samples were transferred to a $60 \mathrm{~mm}$ cell culture dish (on ice) and placed in a Bio- 
LinkTMBLX 365 UV-crosslinker. Samples were crosslinked with UV light at $400 \mathrm{~mJ} / \mathrm{cm} 2$. Samples were then transferred to a fresh $1.5 \mathrm{ml}$ Eppendorf tube. $60 \mathrm{~mm}$ dish was then washed once with $500 \mu \mathrm{l}$ PBS-T and the wash was added to sample. Samples were then centrifuged $8000 \times \mathrm{g}$ at $4{ }^{\circ} \mathrm{C}$ for $5 \mathrm{~min}$. Supernatant was carefully discarded and samples were resuspended in $250 \mu 1-500$ $\mu 1$ RIPA buffer (50mM Tris-HCl pH 7.5, 150 mM NaCl, 1\% Triton-X 100, 0.1\% SDS, 0.1\% Nadeoxycholate, 1mM EDTA, 1x cOmplete, mini EDTA-free protease inhibitor cocktail, RNase inhibitor) and transferred to a Wheaton dounce tissue homogenizer chilled on ice. Samples were homogenized 10x with a loose pestle and then 50x with a tight pestle to ensure complete tissue homogenization. The homogenized samples were transferred to a fresh $1.5 \mathrm{ml}$ Eppendorf tube and kept on ice. The homogenizer was rinsed with $250 \mu 1-500 \mu \mathrm{l}$ IP dilution buffer $(50 \mathrm{mM}$ Tris-HCl $\mathrm{pH} 7.5,150 \mathrm{mM} \mathrm{NaCl}, 1 \mathrm{x}$ cOmplete, mini EDTA-free protease inhibitor cocktail, RNase inhibitor) so as to dilute RIPA buffer 1:1 and the wash solution transferred to same tube as homogenized sample. A 5\% aliquot was taken of the lysate to be used as either input for IP-western blot experiments or input for small RNA sequencing experiment. Sample stored at $-20{ }^{\circ} \mathrm{C}$.

Meanwhile, Diagenode CHiP-kit protein A magnetic beads were washed before use with 500ul PBS-T and then $500 \mu \mathrm{l}$ IP buffer (1:1 RIPA buffer and IP dilution buffer). Samples were precleared with pre-washed $25 \mu \mathrm{l}$ Diagenode CHiP-kit protein A magnetic beads at $4 \mathrm{C}$ with end-toend rotation for $2 \mathrm{hrs}$. A second batch of pre-washed Diagenode CHiP-kit protein A magnetic beads were pre-loaded with rabbit-@-PIWI (ab5207) or IP control rabbit IgG: either $4 \mu$ g antibody for $25 \mu \mathrm{l}$ bead slurry for IP-Western blot experiments or with $8 \mu \mathrm{g}$ antibody for $50 \mu \mathrm{l}$ bead slurry for IP-targeted proteomics experiment/smallRNA library preparation in $500 \mu \mathrm{lP}$ buffer (1:1 RIPA buffer and IP dilution buffer). The bead/antibody solution was incubated at $4{ }^{\circ} \mathrm{C}$ for 4-6 hours with end-to-end rotation. Pre-cleared samples were then pelleted on a magnetic rack and supernatant transferred to the preloaded beads (following the disposal of the supernatant). Antibody/preloaded beads/pre-cleared lysate mixture was incubated at $4{ }^{\circ} \mathrm{C}$ overnight with endto-end rotation. The following day, samples were washed $7 \times 1 \mathrm{ml}$ wash buffer $(50 \mathrm{mM}$ Tris- $\mathrm{HCl}$ $\mathrm{pH} 7.5,150 \mathrm{mM} \mathrm{NaCl}, 2 \mathrm{mM} \mathrm{MgCl} 2,10 \%$ glycerol, 1\% Empigen, RNase inhibitor) ${ }^{34}$. Each wash was carefully removed using vacuum suction. Samples used for IP-western blot or IP-targeted proteomics experiments were eluted with $30 \mu \mathrm{l}$ Bolt LDS sample buffer (Novex, Life Technologies) supplemented with Bolt sample reducing agent (Novex, Life Technologies) at 95 ${ }^{\circ} \mathrm{C}, 1000 \mathrm{rpm}$ for $5 \mathrm{~min}$ then chilled on ice. Samples were stored at $-20{ }^{\circ} \mathrm{C}$ until needed. If RNA was needed following PIWI immunoprecipitation, beads were incubated with $100 \mu \mathrm{TRI}$ Reagent ${ }^{\circledR}$ for 10 min at room temperature. Input samples were incubated with 10 volumes of TRI Reagent ${ }^{\circledR}$ for $10 \mathrm{~min}$ at room temperature. phase lock gel. The tubes were then quickly spun down. Following incubation with TRI Reagent ${ }^{\circledR}$, the beads were pelleted using a magnetic rack and the supernatant was transferred to a tube containing phase lock gel. Subsequently, $20 \mu \mathrm{l}$ chloroform was added, the tubes mixed by shaking and then centrifuged at $3100 \mathrm{x} \mathrm{g}$ for $10 \mathrm{~min}$. The upper phase was transferred to a fresh tube containing $50 \mu \mathrm{l}$ isopropanol and $15 \mu \mathrm{g}$ GlycoBlueTM, mixed by shaking and then incubated at $20{ }^{\circ} \mathrm{C}$ overnight. The samples were then centrifuged at $3100 \mathrm{x}$ g for $10 \mathrm{~min}$ at $4{ }^{\circ} \mathrm{C}$. The RNA pellet 659 was washed with $100 \mu \mathrm{l} 75 \%$ ice cold ethanol, centrifuged at $3100 \mathrm{x} \mathrm{g}$ for $10 \mathrm{~min}$ at $4{ }^{\circ} \mathrm{C}$. 660 Supernatant was carefully decanted off samples and the pellet air dried. The pellet was then 
resuspended in $4 \mu \mathrm{l}$ nuclease free water and stored at $-80{ }^{\circ} \mathrm{C} .5 \%$ of lysate used for PIWI IP (25 $\mu l$ out of $500 \mu \mathrm{l}$ lysate) was reserved to be used as input for small RNA sequencing experiment. These samples were incubated with $250 \mu \mathrm{l}$ TRI Reagent ${ }^{\circledR}$ for $10 \mathrm{~min}$ at room temperature. Subsequently, $50 \mu$ chloroform was added, the tubes mixed by shaking and then centrifuged at $3100 \mathrm{x} g$ for $10 \mathrm{~min}$. The upper phase was transferred to a fresh tube containing $125 \mu$ isopropanol and $15 \mu \mathrm{g}$ GlycoBlueTM, mixed by shaking and then incubated at $-20{ }^{\circ} \mathrm{C}$ overnight. The samples were then centrifuged at $3100 \mathrm{x}$ g for $10 \mathrm{~min}$ at $4{ }^{\circ} \mathrm{C}$. The RNA pellet was washed $1 \mathrm{x}$ with $250 \mu \mathrm{l}$ $75 \%$ ice cold ethanol, centrifuged at $3100 \mathrm{x} \mathrm{g}$ for $10 \mathrm{~min}$ at $4{ }^{\circ} \mathrm{C}$. Supernatant was carefully decanted off samples and the pellet air dried. The pellet was then resuspended in $6.5 \mu$ l nuclease free water and stored at $-80{ }^{\circ} \mathrm{C}$.

IP and Input samples processed the same way from now on: The quality and concentration of the RNA samples was analyzed by Agilent Small RNA kit on the Agilent 2100 Bioanalyzer system and NanoDropTM. The RNA samples were then used to make small RNA libraries using the NEBNext Small RNA Library Prep Kit for Illumina (E7330) according to the manufacturer's instructions. Ligation of the 3 SR adapter was performed at $16{ }^{\circ} \mathrm{C}$ for $18 \mathrm{hrs}$. This longer incubation at a reduced temperature increases ligation efficiency of methylated RNAs such as piRNAs. Since Drosophila RNA is rich in $2 \mathrm{~S}$ rRNA, a $2 \mathrm{~S}$ blocking oligo was also used at a final concentration of $0.1 \mu \mathrm{M}$ to exclude $2 \mathrm{~S}$ RNA from any downstream reaction. 12 PCR cycles were used to amplify the libraries.

684

QC and read mapping

Small RNA reads were trimmed for sequencing adapters using Trim Galore v0.5.0 (https://github.com/FelixKrueger/TrimGalore) in conjunction with Cutadapt v1.11 ${ }^{35}$. Low-quality $(\mathrm{Q}<20)$ bases were also trimmed from the ends of reads, and reads were discarded if their length was then less than 20 nucleotides following trimming.

Trimmed reads greater than 20 nucleotides in length were then mapped to dm6 genome (FlyBase BDGP6.22 release) using the short-read aligner bowtie v1.2.3 ${ }^{36}$, allowing for one mismatch. If there were multiple alignments, then only the highest quality alignment was retained (bowtie options -M 1 --best --strata). Alignment files were sorted and indexed using SAMtools v1.8.

693

\section{Hierarchical Feature Counting}

694

695

Annotation data was downloaded from FlyBase, release BDGP6.22. The annotations were stratified into 14 ordered feature categories: rRNA, tRNA, snRNA, snoRNA, pre miRNA, simple repeats, complex repeats, Piwi RNA, 5' UTR, 3' UTR, protein coding exon, pseudogene, ncRNA, and mitochondrial genome. The Piwi RNA cluster $(n=114)$ annotations were from piPipes. Next, using featureCounts (subread v2.0.0) ${ }^{37}$, mapped reads were counted for each of the 13 categories independently. A minimum overlap of 15 nucleotides was required, with the fractional counts option was on. This counting was strand-specific and was done for both forward (sense) and reverse (antisense) strands independently. Finally, using a custom script (https://github.com/varibbc/Piwi_RNA_pipeline), we did a hierarchical counting for the 13 categories. That is, for any read annotated to multiple features, it was counted only for the feature highest in the category. So, reads mapped to a Piwi RNA cluster within an exon were counted towards the Piwi RNA cluster not the exonic feature. This approach assured that reads were assigned correctly, and ambiguities from reads mapping to rRNA and other small RNA features were not included as Piwi RNAs. This resulted in a counts file for sense and antisense features. 
Differential Expression Analysis

The sense and antisense counts tables were imported into R v3.6.0. Features with counts of less than 10 raw counts in less than 2 samples were removed. In addition, two complex repeat features

711 (LSU-rRNA_Dme and SSU-rRNA_Dme) were also removed. Following filtering, individual 712 contrasts for differential feature expression were done using edgeR v3.28.0 ${ }^{38}$. For all contrasts, a 713 general linear model was fit using appropriate covariates that varied depending on the contrast, but 714 included - where appropriate - treatment, tissue, knock, knock side, and genotype. Statistical 715 significance was assessed using a quasi-likelihood $\mathrm{F}$ test with multiple testing correction 716 performed with the Benjamini-Hochberg procedure.

\section{GSEA}

719 The genes comprising each biotype were used as gene sets to test for enrichment of certain biotypes 720 in certain phenotypes. Enrichment testing was done with GSEA as implemented in the 721 clusterProfiler v3.14.3 function 'GSEA.' Then thousand phenotype permutations were run ${ }^{39}$. The 722 biotype annotation was from UCSC for dm6.

725 ORA for RIP enriched transcripts was performed using WebGestalt ${ }^{31}$ with the following parameters for the enrichment analysis: Minimum number of IDs in the category: 5; Maximum number of IDs in the category: 500; FDR Method: BH; Significance Level: FDR $<0.05$ 